\title{
Tra lingua e lessicografia. Osservazioni su Gli italianismi nel catalano. Dizionario storico-etimologico di Yorick Gomez Gane
}

\author{
Elena Pistolesi \\ Università di Modena e Reggio Emilia \\ elena.pistolesi@unimore.it
}

Il Dizionario curato da Yorik Gomez ${ }^{1}$ colma una vistosa lacuna nella storia delle relazioni fra le lingue romanze, le cui cause aveva riassunto Jordi Bruguera nel capitolo dedicato agli italianismi della Història del lèxic català, ${ }^{2}$ a partire dall'assenza di studi monografici sul tema. Isolare i contributi diretti dell'italiano non è impresa facile. Per la fase più antica (secc. XIII-XIV) la difficoltà principale consiste nel chiarire la procedenza di termini che presentano attestazioni ravvicinate in tutta l'area del Mediterraneo, riconducibili a più volgari parlati sulle sponde della Penisola, dalla Sicilia fino a Genova. Per gli apporti di questi secoli dobbiamo infatti tenere presente che con 'italiano' si rinvia a un variegato contesto plurilingue. E ancora, quando le forme sono attestate in età medievale in tutte le lingue romanze, è arduo fissarne l'origine perché la base può essere, oltre che latina, araba (per es. llebeig, alatxa, sardella) o germanica (per es. foure, estralla, esquiff). Nei secoli successivi (secc. XV-XvI), quando l'italiano si afferma come lingua di cultura europea, si pone il problema delle mediazioni, in primo luogo dal francese e dal castigliano.

Il numero delle parole selezionate da Gomez supera le 500 unità. La stratigrafia degli ingressi è costante dal XıII sec., con rarefazioni che riguardano i periodi di "crisi» delle due culture. L'opera è il risultato di un lavoro accurato e puntuale. Senza entrare nel merito delle singole voci, le note che seguono si concentreranno su alcuni punti critici che possono orientare la consultazione di questo importante strumento.

Come recita il sottotitolo, l'opera è un dizionario storico-etimologico. A questa sintetica descrizione aggiungiamo che si tratta di un dizionario ragionato, con l'organizzazione dei lemmi per campi semantici, all'interno dei quali le voci sono disposte in ordine cronologico in base alla data della prima atte-

1. Yorick Gomez Gane, Gli italianismi nel catalano. Dizionario storico-etimologico, Roma: Aracne, 2012.

2. Barcelona: Enciclopèdia catalana, 1985, p. 78-80. 
stazione. Gli ambiti isolati da Gomez, sulla scorta di Terlingen, ${ }^{3}$ sono: «L'arte e la cultura», «La vita militare», «Il mare», «L'economia», «La vita sociale», «La vita privata». La diversa entità dei prestiti rispetto al castigliano produce vuoti che evidenziano i limiti di questa sovrapposizione: ad esempio, Terlingen (1943) pone nell'area La vida social, al punto H, la Vida del campo, dividendola in a. Términos generales, b. Plantas, c. Animales, ecc. Riprendendo quest'ordine, nella versione di Gomez abbiamo, sotto «La vita sociale», la sequenza: 5.6. "Viaggi e mezzi di trasporto», 5.7. «Flora», 5.8. "Fauna». Il passaggio risulta brusco e, se non si ricorre al modello di riferimento, apparentemente immotivato. Lo stesso avviene in 5.13. dove si legge: «Alla categoria della vita sociale è infine da ricondurre una serie ricchissima di italianismi costituita da lemmi di difficile classificazione, per lo più parole della lingua dell'uso ${ }^{4}$ introdotte tra il XIII e il xx secolo", cui seguono vari paragrafi divisi per categorie grammaticali (verbi, aggettivi, avverbi ecc.), che includono parole spesso contrassegnate come «desuete». Anche la scelta di ordinare le voci basandosi sul terreno delle prime attestazioni, ${ }^{5}$ che è alquanto sdrucciolevole, fa sì che si trattino a distanza parole che sarebbe utile consultare di seguito, come novellar e novella, regatar e regata.

Dai lavori di Terlingen deriva anche lo spoglio proposto in Appendice (Italianismi omologhi in catalano e in castigliano, p. 195-214), che contiene, sempre con riferimento ai paragrafi tematici del volume, le voci attestate anche in castigliano. Questo accorgimento permette di evitare la discussione, che altrimenti sarebbe costante, sui rapporti con il castigliano ed offre un panorama sintetico delle relazioni fra le tre lingue. Non mancano tuttavia, quando necessario, gli approfondimenti nelle singole voci. Gomez afferma comunque che, per i prestiti dall'italiano, il castigliano non sembra avere un peso maggiore del francese. L'osservazione merita di essere sottolineata e potrebbe essere sviluppata tenendo conto della diacronia degli eventuali addensamenti collegati alle vicende politiche e culturali interne alla penisola iberica.

Il lemmario è tratto dallo spoglio dei dizionari catalani di riferimento e da un esiguo numero di studi dedicati al tema. La selezione deriva principalmente dallo spoglio del Diccionari català-valencià-balear di A.M. Alcover e F. de B. Moll $(=$ AlcM), quindi dalla consultazione del Diccionari etimologic i complementari de la llengua catalana di J. Coromines (= DECat) e del Diccionari etimologgic di J. Bruguera (1996), cui si aggiungono il Diccionari de la llengua catalana dell'Institut d'Estudis catalans (= DIccIECat), il Gran Diccionari

3. J.H. Terlingen, Los italianismos en español desde la formación del idioma hasta principios del siglo XVII, Amsterdam: N. V. Noord-Hollandsche Uitgevers Maatschappij, 1943; ID., Italianismos, in Enciclopedia Lingüística Hispánica, diretta da M. Alvar et al., Madrid: Consejo Superior de Investigaciones Científicas, 1967, II, p. 263-306.

4. Il grassetto è nel testo.

5. Segnalo il caso di xarello 'vino rosso chiaro'. Nella discussione si legge "XIV secolo, in Pataffio: GDLI", ma il Pataffio probabilmente risale alla prima metà del secolo successivo (cfr. F. Ageno, «Per l'identificazione dell'autore del Pataffio», Studi di Filologia italiana, xx, 1962, p. 75-84). 
dell'Enciclopèdia Catalana (1998) e il Corpus Textual Informatitzat de la Llengua Catalana (http://ctilc.iec.cat), che contiene materiali dall'Ottocento a oggi, grazie al quale si sono potute retrodatare molte voci, come predel.la, Anunciata, modilló, allegro, andante, fermata, ecc. ${ }^{6}$

Per 'italianismi' si intendono «tout court gli italianismi diretti, tutti quei termini italiani giunti cioè in catalano, con o senza successivi adattamenti morfologici, per via diretta» (p. 10), scritta e orale, attraverso un percorso ritenuto plausibile e verosimile. Per questa ragione sono stati opportunamente esclusi gli italianismi occasionali, creati o usati da un solo autore (si cita in merito la traduzione della Commedia di Andreu Febrer), anche se alcuni casi dubbi restano nelle maglie della selezione (enrosir, nitxo, ecc.). Qualche problema pone la selezione dei toponimi. Che Liorna sia il nome catalano (e castigliano) di Livorno è un caso ben diverso da quello di anxova, tanto per fare un esempio. Infatti Liorna, che Gomez include come «italianismo secondo $A l c M$ », risulta nella fonte citata semplicemente: "Ciutat de Toscana, port de mar, anomenada en italià Livorno; cast. Liorna». In tal senso, si sarebbe potuta includere qualunque città, come Génova («Etim.: de l'it. Genova (en llatí Genüa), nom de la dita ciutat»).

I limiti delle fonti, con al centro AlcM, si riflettono nella selezione del lemmario. Un dizionario è fatto per la consultazione puntuale, non per una lettura integrale, come quella che tocca al recensore, dalla quale emergono però alcune particolarità, come la tendenza dell' $A l c M$ ad accreditare, da solo, come italianismi parole che altre fonti non segnalano come tali o per le quali ipotizzano provenienze diverse. Tale concentrazione desta qualche sospetto sull'impianto complessivo dell'opera di riferimento. Senza entrare nella polemica sul mozarabico, che ne ispirava le parole, Coromines osservava che "per a AlcM l'italià és la panacea etimològica».?

Le riserve di Coromines sembrano confermate dalle voci attestate prima in catalano che in italiano, che l'AlcM considera da solo italianismi, tra le quali, per limitarci ad alcuni esempi tratti dal vocabolario del mare, troviamo: amainar 'ammainare', andana 'serie di barche ormeggiate alla banchina una di

6. Avrei inserito nella bibliografia il Glossari general lul.lià di Miquel Colom (Palma de Mallorca: Editorial Moll, 5 vol., 1982-1985), che attinge anche a fonti inedite. Per gli studi futuri sarà possibile ricorrere al Diccionari de textos catalans antics (http://www.ub.edu/ diccionari-dtca/index.php), che consente già oggi la consultazione di un numero consistente di testi.

7. Cfr. G. Colón Domènech, «Francesc De B. Moll i l'etimologia», in Francesc De B. Moll a l'inici del segle XXI, a cura di Maria Pilar Perea, Barcelona: UB, 2003, p. 23-29 (a p. 26, n. 3). Riporto di seguito alcune voci, limitate al campo dell'economia, che l'AlcM attesta da solo o che da solo indica come italianismi contro altre ipotesi: rescatar 'riascattare' duquena 'ufficio fiscale', crèdit 'scambio d'un bene attualmente disponibile con una promessa di pagamento', saldo 'saldato', acaparrar 'fissare un acquisto dando caparra', bancarota 'mancato pagamento dei propri debiti da parte di un imprenditore', agio 'maggior valore ... ottenuto nel cambio di moneta', testó 'nome di moneta', piastra 'moneta d'argento', manyà 'fabbro', marçapà 'scatola speciale contenente prodotti di farmacia o dolci', lambart 'muratore o capomastro', porcellana 'materiale ceramico', ecc. 
fianco all'altra', badafió 'piccolo cavo che serve a sostenere la vela', bergantí 'brigantino', brúixola 'bussola', galaverna 'fasciatura del ginocchio del remo', galera 'nave da guerra a remi o a vela', galiot 'rematore di galea', pasteca 'bozzello con apertura laterale per il passaggio di una fune', remolar 'fabbricante o riparatore di remi', romball 'tassello di legno di forma romboidale usato per riparare buchi nel fasciame', tonaira 'tonnara', trossa 'collegamento a collare che unisce all'albero la parte centrale del pennone'. L'entità dei casi dubbi, che sarebbe stato utile riunire in un'appendice a sé, riduce considerevolmente il numero complessivo dei prestiti diretti, certi o verosimili.

Talvolta la prima attestazione catalana può servire a retrodatare quella italiana (cfr. saquejar 'saccheggiare', o coronel 'colonnello'). In merito a pavès 'scudo rettangolare' si legge: "la forma catalana (senza $n$ ) può servire a retrodatare la forma italiana senza $n$ dal 1342 al 1309». La nota poteva essere corretta consultando il Tesoro della Lingua Italiana delle Origini (http://tlio. ovi.cnr.it/TLIO/) s.v. pavese, dove si riportano le diverse forme della parola (palvese, pavese, panvese, ecc.) e nel quale la prima attestazione (senza $n$ ) nell'accezione di 'scudo' risale al 1303, mentre con il valore di 'fortificazione' si trova già nel 1288. Sempre al TLIO si poteva ricorrere per la voce Gatzaro 'eretico, in particolare della setta dei Catari', che si conclude con «Dall'italiano secondo AlcM, che cita però la forma gazzaro, non attestata». La parola è attestata, così come l'espressione gazzaro patarino 'sodomita', che avrebbe giovato alla complessa discussione sull'etimo proposta dall'autore.

Il Dizionario nasce come parte di un progetto più ampio sugli italianismi in tutte le lingue del mondo, che poi è stato accantonato. ${ }^{8}$ La collocazione di questo lavoro entro il quadro europeo avrebbe probabilmente chiarito molti dubbi sull'adozione diretta e indiretta delle parole. Si ha l'impressione che la struttura italianocentrica dell'opera sia un'eredità del disegno iniziale. Le voci si aprono, di solito, con l'indicazione tratta dal GDIU (= T. De Mauro, Grande dizionario italiano dell'uso, 1999-2007) relativa alla marca d'uso (fondamentale, comune, tecnico, ecc.), e con il rinvio al DELI (= M. Cortelazzo / P. Zolli, Dizionario etimologico della lingua italiana, Bologna: Zanichelli, 1999) per l'etimo e la prima attestazione. Si avverte la mancanza di una valutazione analoga a quella fornita dal GDIU nella lingua di arrivo (per intenderci: mandra non ha in catalano lo stesso peso di malandri), che si sarebbe potuta derivare, almeno parzialmente, dal DIccIECat. Solo in modo sporadico compare l'indicazione «desueto» (etichetta applicata ai lemmi "solo antichi, mancando nei lessici dell'uso»).

L'indicazione delle marche d'uso del GDIU appare inutile quando si affiancano accezioni molto diverse nelle due lingue. Per esempio, sotto la voce catalana $t u t i^{2}$ 'gioco di carte', si legge: «tra i “vocaboli fondamentali” secondo

8. Per la presentazione dell'iniziativa si può legge L. SERIANNI, «Gli italianismi nelle altre lingue romanze: prime riflessioni», in Italianismi e percorsi dell'italiano nelle lingue latine (Atti del Convegno di Treviso 28 settembre 2007), Parigi: Unione Latina, 2008, p. 19-41 (http://www.unilat.org/data/publications/75.pdf). 
GDIU» con riferimento all'italiano tutto, ma «in italiano non sembra attestato (almeno in ambito lessicografico) un nome di carte del genere». Qualche incoerenza si osserva nella struttura delle voci: salta all'occhio, proprio per il suo carattere programmatico, la sporadica assenza del riferimento al GDIU (cfr. sonet, Manierisme, manierista, bambotxada, concert, ecc.); poco calibrato è poi lo spazio riservato all'etimologia, che oscilla tra discussioni impegnative e riproduzione integrale delle fonti $(D E L I)$. Il secondo caso è esemplificato dal tuti appena citato, dove, a distanza ravvicinata, troviamo riprodotta la stessa lunga nota etimologica sotto tuti ( ${ }^{1}$ (ututi 'azione svolta dalla totalità dei presenti'») e tuti ('gioco di carte') senza che questa chiarisca alcunché sulla natura dei prestiti.

I problemi di struttura delle voci non inficiano il valore complessivo del Dizionario, che costituirà un punto di riferimento imprescindibile per i futuri studi sulle relazioni tra italiano e catalano. La selezione accurata dei lemmi indica infatti la strada da percorrere per accertare i casi dubbi e per filtrare le potenziali nuove entrate. 